\title{
Research and Survey on the Status of Hospital Graded Care System and its Related Industries
}

\author{
Weijie Tang ${ }^{1}$ \\ ${ }^{1}$ School of Management, Wuhan University of Technology, Wuhan, China
}

\begin{abstract}
The graded treatment system refers to the gradation of diseases according to their priority and ease of treatment, with medical institutions at different levels undertaking the treatment of different diseases and gradually realizing the medical process from general practice to specialization. Since the "new medical reform", China has been committed to promoting "primary care", "two-way referral", "separation of acute and slow treatment "The "new health care reform" has been implemented in China since the beginning of the reform. However, in the process of implementation, the effectiveness of the system has always been controversial due to the inadequate construction of primary medical institutions and the weak awareness of graded treatment among residents.
\end{abstract}

\section{Significance of the study}

\subsection{Theoretical implications}

This study brings two theoretical innovations to research related to the hierarchical system.

First, a new perspective on the evaluation of the tiered medical treatment system is proposed. This study analyzes and evaluates the implementation of the tiered medical treatment system from the perspective of patients' perceived value and satisfaction, providing new ideas and directions for the evaluation of the tiered medical treatment system.

Secondly, the evaluation system of patients' satisfaction with graded medical treatment was constructed. Based on the SOR theory model, the CSI Asian Customer Satisfaction Model and the customer transfer value theory, this study constructs an evaluation system for patient satisfaction surveys under the tiered treatment system.

\subsection{Practical implications}

As a reflection on the health care system in society, this study can help the authorities, the health care system, the health care providers and other institutions to provide health care services with a higher level of satisfaction to patients.

First, the theory guides those in power to reform and optimise the hierarchical medical system, and drives the relevant referral and medical reimbursement systems to synchronise with it, while helping to rationalise the distribution of health resources, solving the problem of wasted resources caused by the fact that higher-level hospitals with high-quality resources are taking on a large number of general outpatient tasks, and at the same time leading to an increase in the utilisation of primary medical resources.

\section{Current status of research}

\subsection{Current status of domestic research}

Since the founding of the country, there have been regulations on the hierarchical diagnosis and treatment system in China, but no complete system has been formed. Since the new medical reform in 2009, various provinces and cities have been exploring the construction path of the hierarchical diagnosis and treatment system, and the construction of the hierarchical diagnosis and treatment system has been put on the important agenda of medical reform, which has gradually led to the system of hierarchical diagnosis and treatment system. The number of studies on graded diagnosis and treatment has been concentrated since that time and has shown a trend of continuous growth.

In the five years since the new medical reform, scholars have focused their research on the implementation and execution of graded treatment. Yao $\mathrm{Ke}$ put forward the main difficulties in the implementation of the tiered diagnosis and treatment system: firstly, the weakness of the bottom of the primary medical service network; secondly, the difficulty in achieving orderly triage; and thirdly, the backwardness of medical information technology construction. And then, corresponding development directions and specific measures were proposed, and 
these key findings were also closely followed by experts and scholars in the subsequent studies. However, as this stage was at the early stage of the reform of the construction of the grading and treatment system, scholars did not understand the specific problems in the implementation of the grading and treatment system, and fewer suggestions with feasibility were put forward.

Immediately afterwards, domestic research on the tiered diagnosis and treatment system focused on the system and its measures to support tiered diagnosis and treatment. Zhang Fan mentioned that the current problem with the implementation of graded treatment is that "it is easy to transfer up but difficult to transfer down". Research shows that to promote the system of graded diagnosis and treatment, it is necessary to promote comprehensive hospital reform, establish supporting policies, improve the macroeconomic control mechanism of medical resources, train a team of grassroots doctors and establish an information sharing mechanism. At this stage, a large number of studies have pointed out the difficulties of "downward referrals" in the implementation of the hierarchical diagnosis and treatment system and the importance of building up primary medical resources, but the conclusions and recommendations mostly remain in the policy itself.

\subsection{Status of foreign research}

The use of the graded care system is widespread in developed countries, but its use in China is not promising. Overseas research has focused on two main areas. One is the study of public perceptions of the tiered care system. liu Xiaojun et al. conducted a crosssectional study of tiered care patients and showed that gender, age, education level and place of residence influenced the level of public perceptions of the tiered care system (NHMS). in 2019 Yan Yu-Hua et al. studied the impact of the tiered care system on the public's healthcare seeking behaviour also found that there were significant differences in the perceptions of the new policy by age, marital status, annual income, education level and occupation. guihao Liu et al. used pregnant women as the survey population to investigate the choices made when seeking medical treatment, and selected several hospitals to conduct questionnaires on groups of pregnant women. the results of the survey fully affirmed that the tiered treatment system had played a positive role in the regulation of medical resources, but there were still The results of the survey confirmed that the tiered system has had a positive effect on the regulation of health care resources, but that there is still "overstepping of medical resources", for example, highly educated pregnant women who are not at greater risk still overstepping medical resources.

Peijia Ren and Zhang Runtong used the intuitionistic fuzzy number MCDM thermodynamic method and the image focal point-choquet integral aggregation operator, respectively, to optimize the decision making model. The optimization of the decision model was carried out. The above studies have answered the two most important stages of patient access in the hierarchical diagnosis and treatment system: the improvement paths of system cognition and decision making, but neglected to evaluate and improve the hierarchical diagnosis and treatment system from the public's perspective. Moreover, the large population base in China and the obvious differences among citizens make the studies of hierarchical diagnosis and treatment systems in foreign regions may not be able to describe the domestic situation well. This study attempts to examine the tiered treatment system from the perspective of public satisfaction.

\section{Theoretical foundations}

\subsection{What's innovative}

(1) Use the rooting theory to distil inductively the various factors that influence patient satisfaction.

(2) Using SOR theory to construct a structural equation for the factors influencing satisfaction, based on consideration of moderating variables

Explore the relationship between satisfaction and variables.

(3) Establishing patient satisfaction based on the CCSI theory model and the customer transfer value model, based on the actual situation Degree evaluation system.

\subsection{SOR theoretical model}

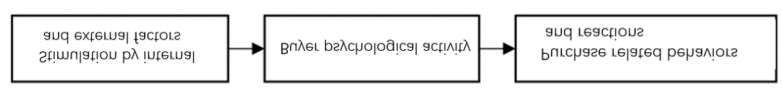

Figure 1 - SOR theoretical model diagram

The general model of human behaviour is the S-O-R model, i.e. 'stimulus - individual physiological, psychological - response'. The application of this model to the present study suggests that patients' consultation behaviour is stimulated by stimuli that arise from both their objective need for physical illness and their subjective judgement based on external information and their own psychology. Patients are motivated by these factors to seek treatment. This motivation leads to a decision to seek treatment and to the act of seeking treatment. After the act, an evaluation of the process and the outcome of the consultation is made, which completes the consultation process.

\subsection{Theory of customer transfer value}

Definition of customer transfer value: The actual value transferred by the company and felt by the customer. It is generally expressed as the difference between the total value of the customer's purchase and the total cost of the customer's purchase. It is considered that the total customer cost consists of monetary cost, time cost, energy cost and physical cost, while the total customer value consists of product value, service value, personnel value and image value. 


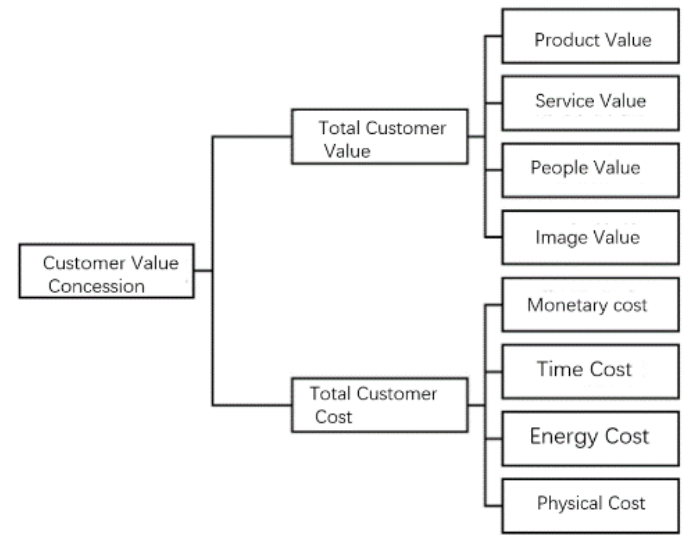

Figure 2 Theoretical diagram of the value of customer concessions

In this study, satisfaction was estimated by substituting the value of concessions for satisfaction and thus patient satisfaction during the NCCP. The indicators were categorised as hospital class, service experience, doctor's level, institutional reputation; cost of visit, waiting time, choice of energy, and stamina of consultation were designed as factors, research hypotheses were made and surveyed for validation.

\subsection{KANO model}

The KANO model (Kano model) is a useful tool for classifying and prioritising user needs, based on an analysis of the impact of user needs on user satisfaction, reflecting the non-linear relationship between product performance and user satisfaction. In the Carnot model, the quality characteristics of products and services are classified into four types: (1) essential attributes, (2) desired (linear) attributes, (3) charismatic attributes and (4) undifferentiated attributes. and are influenced by inverse factors, such as: time, cost, etc.

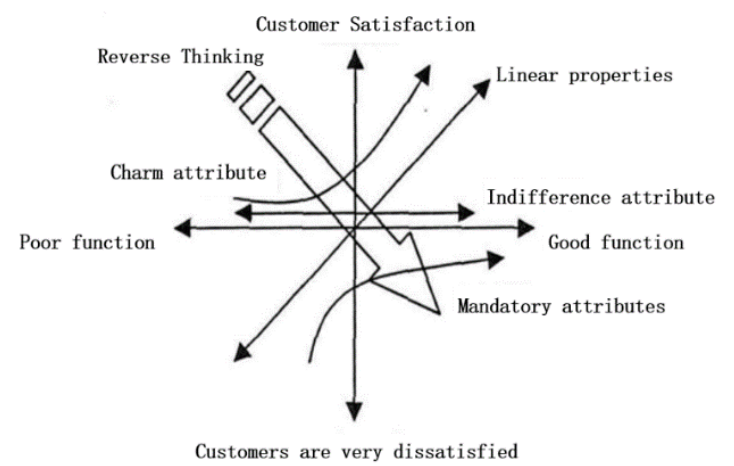

Figure 3 - KANO model

This study uses the KANO model to analyse the priority ranking of factors affecting patient satisfaction and to find the best way to improve patient satisfaction with the hierarchical medical system.

\section{Research programme}

\subsection{Research Methodology}

The literature review method: finding relevant literature (journals, papers, books, etc.) through a variety of sources adds persuasive value and provides strong evidence for the conduct of the project.

Likert scale method: online as well as paper questionnaires were designed, distributed, collected and used to obtain authentic data from a variety of sources, with those who had visited the clinic during the epidemic as the main respondents to find out their satisfaction.

In-depth interview method: Through communication with representative patients, doctors, etc., we can learn more and deeper about satisfaction.

Field survey method: The field survey is used to understand the patient's visit to the three levels of hospital to get a clearer picture of their real situation.

Data analysis methods: Correspondence analysis, cross-group analysis, cluster analysis and factor analysis using SPSS 18.0, SASS.2 was used to do partial least squares analysis.

\subsection{Technical routes}

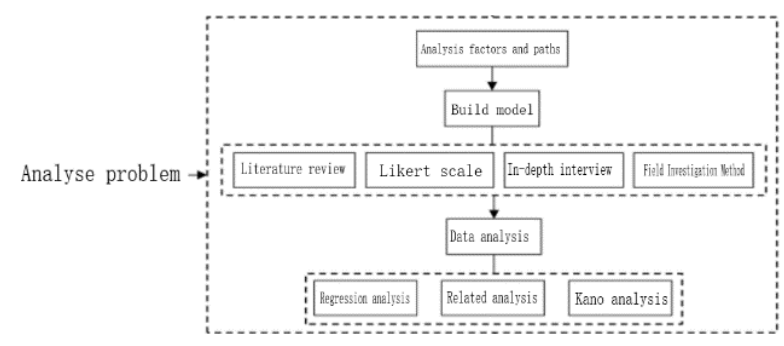

Figure 4 Technical routes

\section{Research feasibility}

\subsection{Need for a research project}

The lack of proper application of the hierarchical system has led to patients neglecting primary care and flocking to secondary and tertiary hospitals, resulting in varying degrees of NCCP patients being denied efficient treatment due to the "difficulty of access" and delaying treatment. This has resulted in a vicious circle of patients who are unable to receive efficient treatment because of the difficulty in accessing medical care, delaying the best time for treatment and causing serious consequences, or causing cross-infection and increasing the number of patients. This project provides advice and recommendations on the practical problems of stratified medical care, providing a theoretical basis for optimising and upgrading the hierarchical medical treatment system, and effectively solving patients' treatment problems. 


\subsection{Technical feasibility}

The project relies on the customer transfer value theory to analyse the factors and the CSI satisfaction model to build a satisfaction evaluation system for the tiered medical care system. The study uses the SOR theoretical model to help investigate the nature of the phenomenon that a large number of undiagnosed patients ignore the graded care system during the New Coronation epidemic, leading to a lack of medical resources in tertiary hospitals, by exploring the objective needs of patients for medical treatment as well as the stimulus of external environmental and internal psychological factors. Within the existing technical framework, the data collected were statistically collated and analysed using SPSS analysis, and a number of hypotheses were tested using Likert scales to determine the factors of patient satisfaction with the tiered system and to construct a satisfaction evaluation system. It is technically feasible, both in terms of tool selection and theoretical construction, as it can help us to solve a series of problems faced in the project.

\section{References}

1. Xu ZW, Zhang J, Qin CY, Jia LY. Current situation and policy analysis of the two-way referral system for graded care [J]. Hospital Management Forum, 2018, 35(03): 11-14.

2. Liang Yan. Seizing the opportunity of "epidemic" to speed up the implementation of graded diagnosis and treatment $[\mathrm{N}]$. Changzhou Daily, 2020-0302(A04).

3. Chen Ruiying. The historical evolution and development path of China's graded diagnosis and treatment system[J]. Journal of Liaoning Medical College (Social Science Edition), 2016, 14(03)

4. Yao K. A few suggestions for vigorously promoting graded diagnosis and treatment and two-way referral system[J]. Qianjin Forum, 2014(04) 